\title{
Algunas cuestiones relevantes en la enseñanza de las ciencias desde una perspectiva Ciencia-Tecnología- Sociedad
}

\author{
Teresa Prieto, Enrique España y Carolina Martín \\ Facultad de Ciencias de la Educación. Universidad de Málaga.España.ruz@@uma.es
}

[Recibido en mayo de 2011, aceptado en septiembre de 2011]

\begin{abstract}
En este artículo se analizan algunas de las respuestas que se dan en la actualidad a tres preguntas clásicas en la enseñanza de las ciencias: por qué enseñar ciencias, qué ciencia enseñar, y cómo enseñarla, desde una perspectiva Ciencia-Tecnología-Sociedad (CTS). Se argumenta sobre la necesidad de preparar a los futuros ciudadanos para tomar decisiones responsables en asuntos relacionados con la ciencia y la tecnología en la sociedad del siglo XXI, y la conveniencia de utilizar problemas socio-científicos en el aula de ciencias. Finalmente, el análisis es ejemplificado en dos casos: el consumo de alimentos y el consumo de energía.
\end{abstract}

Palabras clave: Alfabetización científica y tecnológica; toma de decisiones; Ciencia-Tecnología-Sociedad (CTS); problemas socio-científicos; consumo de alimentos; consumo de energía.

\section{Some relevant questions in science education from the perspective Science- Technology-Society}

In this article, some of the answers given at this time to three classic questions related to science teaching: why teach science?, what kind of science to teach?, and how to teach it?, are analyzed from a Science-TechnologySociety perspective (STS). It argues for the need to prepare future citizens to make responsible decisions on matters related to science and technology in the XXI century, and the convenience of using socio-scientific issues in the science classroom. Finally, the analysis is exemplified in two cases: food consumption and energy consumption.

Keywords: Scientific and technological literacy; decision making; Science-Technology-Society (STS); socio-scientific issues; food consumption; energy consumption.

\section{Introducción}

En la Didáctica de las Ciencias existen tres preguntas clásicas y siempre vigentes: ¿por qué enseñar ciencias?, ¿qué ciencia enseñar? y ¿cómo enseñarla? (Lucas, 1993). Las respuestas a estas preguntas están estrechamente relacionadas. Así, las razones por las que enseñamos ciencias inciden en la ciencia que enseñamos y en cómo lo hacemos. Ahora bien, si las preguntas son tan clásicas como actuales, las respuestas varían con el momento histórico y el contexto social.

En las últimas décadas, los espectaculares avances científicos y tecnológicos han transformado no solo nuestras vidas, sino también las estructuras de la realidad social, política y cultural. Nuestra sociedad ha pasado de la era industrial a la era del conocimiento y de la información. Colucci-Gray, Camino, Barbiero y Gray (2006) se refieren a las numerosas manifestaciones del poder que tiene la tecnociencia para transformar los sistemas naturales y sociales a escala global, y se plantean la necesidad de una profunda reflexión acerca de los seres vivos, su dependencia del medio natural y la responsabilidad del ser humano sobre el planeta.

Teniendo en cuenta la realidad antes referida, en este artículo analizamos algunas respuestas actuales a las tres preguntas planteadas, desde un enfoque Ciencia-Tecnología-Sociedad (CTS). 


\section{¿Por qué enseñar ciencias?}

Desde la Didáctica de las Ciencias, las propuestas de alfabetización científica y tecnológica hacen referencia a la necesidad de que todos los ciudadanos reciban una formación integral que les capacite para ejercer plenamente sus derechos e intervenir en los procesos de toma de decisiones que se dan en las sociedades democráticas actuales. Porque, si la mayoría de la población se siente ajena a este conocimiento e incapaz de comprender cuestiones actuales de gran trascendencia, podría desentenderse de ellas, y dejaría en otras instancias la responsabilidad que le corresponde. Por ello, los procesos de toma de decisiones están adquiriendo un protagonismo cada vez mayor en la enseñanza de las ciencias. Las personas nos vemos a diario en situaciones en las que debemos tomar decisiones en una gran variedad de asuntos muy relacionados con la ciencia y la tecnología en el contexto de problemas de gran actualidad y relevancia, y cuyas repercusiones no solo nos afectan individualmente, sino también a nivel global.

En la sociedad actual resulta imprescindible contar con una ciudadanía científica y tecnológicamente alfabetizada por razones de diferente tipo: de carácter económico, políticosocial, cultural y funcional (Fourez, 1994; Millar, 1996; DeBoer, 2000; Ryder, 2001; Hodson, 2003; Bybee y Fuchs, 2006).

Las razones de caracter económico están basadas en la convicción de que ningún país podrá asegurarse un desarrollo continuo y a largo plazo si no se llevan a cabo programas educativos dirigidos, tanto a mejorar la alfabetización científica del conjunto de la población, como a la preparación de científicos y tecnólogos.

En estrecha relación con estas últimas, están las razones de carácter político-social, fundamentadas en la percepción de que, si la ciudadanía no posee la cultura científica y tecnológica suficiente que le permita comprender y controlar democráticamente las decisiones de científicos y tecnólogos, los sistemas democráticos se harán cada vez más vulnerables a la tecnocracia.

Las razones de carácter cultural apelan al derecho de cada ser humano a disfrutar del conocimiento científico-tecnológico y a la conveniencia de considerar a la ciencia y la tecnología como parte de la cultura en la que nos hallamos inmersos.

Por último, y como consecuencia de la presencia creciente de la tecnociencia en nuestro mundo, surge el carácter funcional de este conocimiento. Un nivel adecuado del mismo se considera imprescindible para que podamos desenvolvernos sin dificultades en el mundo que nos rodea, entendiendo los sucesos que en él se producen y actuando sobre ellos en la medida de nuestra responsabilidad.

En suma, estar alfabetizado científica y tecnológicamente abre la puerta a habilidades y competencias que contribuyen al empoderamiento de la ciudadanía para la toma de decisiones sobre problemas que inciden en sus vidas (Schreiner, Henriksen y Hansen, 2005). Este empoderamiento va más allá de la capacidad para comprender los problemas, y requiere la voluntad de implicarse en ellos, desarrollando una actuación responsable en el contexto en el que a cada uno vive.

Es necesario, pues, ampliar la perspectiva más clásica de la enseñanza de las ciencias, lo cual, como señalan Ratcliffe y Millar (2009), requiere cambios importantes que tienen implicaciones en la selección de los contenidos, en su estructuración y en la forma en que se trabajen en el aula. 


\section{¿Qué ciencia enseñar?}

Nos encontramos ante un concepto de alfabetización científica y tecnológica que ha ido creciendo y evolucionando a lo largo de los años (DeBoer, 2000). Ahora, frente a formulaciones anteriores, se puede decir que ciencia y tecnología resultan cada vez más difícil de separar. Jenkins (2009) enfatiza, por un lado, la necesidad de considerarlas como dos sistemas que interaccionan intelectual y socialmente $y$, por otro, la necesidad de seleccionar problemas y ejemplos que pongan de manifiesto esta realidad.

Hodson (2003) se refiere a la conveniencia de "politizar" el currículum de ciencias y de tecnología en el sentido de que incluyan el análisis de algunos de los desafíos que preocupan a la humanidad en la actualidad, como elemento formativo para la acción responsable.

En definitiva, estos planteamientos muestran la importancia de proporcionar una formación científica y tecnológica que capacite a nuestros estudiantes para la toma responsable de decisiones en la sociedad del siglo XXI. Para ello, parece necesario hacer un enfoque CTS en el ámbito de la educación científica, de manera que los contextos científico, tecnológico y social implicados en cada dominio sean identificados y analizados de manera conjunta. Es un cambio que exige interdisciplinaridad en los contenidos, integración de conocimientos procedentes de los contextos académicos y sociales, y el análisis de los problemas actuales que inciden en el futuro de la sociedad. Dentro de este marco interdisciplinar y de integración, no podemos olvidar los objetivos que se persiguen en la enseñaza de las ciencias y que deben ser el pilar principal a la hora de seleccionar contenidos (DeBoer, 2000). En esta línea, se pueden destacar cuatro dimensiones que ayudan a la selección de los contenidos:

Una primera dimensión de tipo práctico, relativa, por una parte, a los conocimientos útiles en diferentes profesiones (por ejemplo, electricista o fontanero) y, por otra, a situaciones cotidianas que requieren tomar decisiones que afectan a nuestro bienestar (por ejemplo, elaborar una dieta equilibrada o elegir productos de limpieza en el hogar). Igualmente práctico resulta estar familiarizado con numerosas aplicaciones tecnológicas, tan importantes como útiles hoy en día.

Una segunda dimensión se refiere al conocimiento que nos lleva a comprender problemas sobre los que también tomamos decisiones con frecuencia y que tienen que ver con nuestra responsabilidad social (por ejemplo, las razones para no utilizar determinados productos dañinos para la capa de ozono, las implicaciones del consumo excesivo de energía, o cuestiones relativas a los alimentos modificados genéticamente).

A la tercera dimensión la podríamos denominar "enseñar a pensar". Aprender, en esta dimensión, tiene que ver con la educación del pensamiento, en el sentido de hacerlo más sistemático y racional. Los estudiantes deben ser capaces de reconocer los procedimientos científicos, diferenciarlos de los que no lo son y llevarlos a la vida cotidiana mediante conceptos como: validez, evidencias, objetividad, incertidumbre, asunciones, etc. Al mismo tiempo, deben reconocer los límites de la ciencia con otras formas de pensamiento.

Por último, una dimensión relativa a la ciencia como un saber en el mundo que, junto con la tecnología, forma parte de la herencia cultural que se transmite de generación en generación. En ella cobran relevancia, tanto la trascendencia histórica de algunas teorías científicas o el desarrollo de algunas tecnologías, como la consideración de cómo inciden ambas en la configuración de la sociedad. 


\section{¿Cómo enseñar ciencias?}

Con relación al cómo enseñar, en coherencia con lo expuesto anteriormente, resulta conveniente partir de situaciones de la vida real y diseñar, en torno a ellas, actividades que ofrezcan oportunidades para el desarrollo de los aprendizajes que deseamos promover. Es decir, oportunidades para formular problemas, seleccionar información relevante y analizarla, desarrollar los conceptos científicos implicados, la formulación de hipótesis y conclusiones, etc. Cuando nos centramos en problemas reales aumentan las ocasiones de poner en juego las actitudes y valores que afloran en los contextos y procesos de toma de decisiones.

Al tipo de problemas que tienen el potencial de aunar una naturaleza CTS con una alta implicación de los valores y la ética, se les suele denominar "problemas socio-científicos" (España y Prieto, 2009 y 2010). La controversia que les acompaña permite aportar contextos para el debate y la búsqueda de soluciones y de acción (Zeidler y Sadler, 2008; Prieto y España, 2010).

A continuación se presentan dos ejemplos de los problemas a los que estamos aludiendo. Ambos, aunque diferentes, tienen que ver con el consumo en la sociedad actual: de alimentos y de energía.

\section{El consumo de alimentos}

Los alimentos y la alimentación tienen gran importancia en nuestras vidas. Sobre ellos hemos de tomar decisiones cotidianas que tendrán claras repercusiones en nuestra salud. Por otra parte, a través de los medios de comunicación, que tan importante papel juegan en la sociedad de la información, nos llegan noticias sobre acontecimientos como: el envenenamiento por aceite de colza, el llamado "mal de las vacas locas", la polémica sobre los alimentos modificados genéticamente, etc.

El problema de lo alimentos modificados genéticamente nos ilustra como la biotecnología y sus aspectos controvertidos están en el centro del debate social. Este debate ha evolucionado mucho, desde las primeras críticas en las que se ponía de manifiesto la preocupación por el hecho de que el ser humano pudiera intervenir sobre el genoma, pasando por las críticas centradas en la intervención sobre animales y el riesgo de introducir cultivos modificados genéticamente en el ambiente. En los últimos años, las discusiones se han centrado en los efectos de los alimentos modificados genéticamente sobre la salud humana y el medioambiente (Muñoz, 2002).

Por lo tanto, podemos llevar los alimentos modificados genéticamente al aula como problema socio-científico, dada la controversia en la que se encuentran envueltos y su contextualización en la vida diaria. Para ello, actividades como los debates o los juegos de rol resultan muy adecuadas (Simonneaux, 2001). En esta línea, un ejemplo de actividad sería el juego de rol "juicio a los alimentos transgénicos" (España, Prieto y González, 2004) diseñado para alumnos de tercero de ESO en una unidad didáctica sobre los alimentos y la alimentación. En este tipo de actividades es esencial la búsqueda de información veraz por parte de los alumos/as para fundamentar con datos y pruebas los propios argumentos y confrontarlos con los de otros, en procesos que favorecen el pensamiento reflexivo y la toma responsable de decisiones. Además del conocimiento científico sobre este tipo de alimentos, en la actividad también se ponen en juego valores y actitudes, tan importantes en la toma de decisiones sobre relacionadas con el consumo (España, 2008). 


\section{El consumo de energía}

La producción y el consumo de energía es otra cuestión de gran transcendencia hoy en día. Es un hecho que el consumo de energía en las sociedades avanzadas no deja de aumentar, hasta el punto de que se suele calificar a nuestras sociedades como "energívoras". Por ello, se plantea la necesidad de buscar un modelo de consumo de recursos energéticos que sea sostenible tanto para el ser humano como para el medioambiente y pueda satisfacer las demandas productivas de la sociedad actual y futura.

Es un problema global que requiere que todos los ciudadanos tomen decisiones en su ámbito más personal, para frenar la dependencia actual de determinadas fuentes de energía en favor de otras con menos implicaciones mediambientales, económicas y políticas.

Los últimos sucesos acontecidos en Japón o en la guerra de Libia son una muestra de la necesidad de analizar y reflexionar sobre los diversos factores que conforman el problema (Martín y Prieto, 2010). Por ejemplo, se pueden destacar factores sociales, asociados a la preocupación por la supervivencia de nuestro nivel de bienestar; factores medioambientales, ligados al impacto que tiene en el medio natural el uso de determinadas fuentes de energía, como los combustibles fósiles o los componentes radiactivos (artífices de la energía nuclear); o factores económicos y políticos, ligados al elevado coste que el ciudadano tiene que pagar por el disfrute de los mismos, asociado en la mayoría de las ocasiones a los intereses políticos de determinados países.

Por tanto, el consumo de energía es un tema de gran controversia y que puede considerarse como un problema socio-científico que nos involucra y afecta a todos y en cuyo tratamiento en el aula se implica, como en pocos, el esfuerzo de interdisciplinaridad que anteriormente hemos considerado tan necesario (Martín, y Prieto, 2011). Será imprescindible que el alumnado se plantee cuál es la procedencia de la energía que consumimos o que reflexione sobre cuáles son sus hábitos relacionados con el consumo energético. Para ello proponemos la realización de actividades en torno a situaciones de su vida en las que, por ejemplo, los alumnos/as se vean en la necesidad de calcular su contribución a la emisión de dióxido de carbono al medioambiente, a partir del análisis de su propio consumo energético individual, o de identificar de dónde procede la energía que llega a sus casas.

Este sería el punto de partida para trabajar un problema con un alto potencial educativo, ya que además de implicar al aprendizaje conceptual relacionado con la energía, se ven involucradas las actitudes, los valores, los comportamientos y la conciencia de las personas. Todos estos elementos nos colocan en el camino de promover el empoderamiento, tan necesario para facilitar la toma de decisiones de la ciudadanía en torno a la producción y el consumo de energía.

\section{Consideraciones finales}

La enseñanza de las ciencias constituye un vehículo privilegiado para permitir que los futuros ciudadanos se integren en una sociedad cada vez más tecnificada, e inmersa en continuos cambios. Por ello, creemos necesario avanzar hacia perspectivas más integradoras que acerquen al alumnado a la realidad en aspectos relacionados con la ciencia y la tecnología y que permitan, tanto al alumnado como al profesorado, percibir el sentido, el beneficio y la utilidad que les proporcionan. Es decir, tenemos que buscar vías para: a) enfatizar la interrelación entre la ciencia y la tecnología para llevar a la práctica una integración explícita de las mismas; b) ampliar esta integración con los aspectos sociales; c) atender al desarrollo de competencias para gestionar la información: pensamiento crítico, selección de información, análisis y 
síntesis, comunicación; etc.; d) enlazar con la motivación de la persona a través de los valores, la ética y la responsabilidad; etc.

Nos hace falta, a la comunidad de enseñanza de las ciencias, un dialogo honesto sobre el por qué, el qué y el cómo enseñar ciencias, y ver la forma de ponernos de acuerdo sobre lo que hay que hacer para que los alumnos/as encuentren interesante lo que aprenden y les motive para continuar aprendiendo, haciéndose cargo de su importancia al tomar decisiones en sus vidas.

\section{Referencias}

Bybee, R. W. y Fuchs, B. (2006). Preparing the 21st Century Workforce: A New Reform in Science and Technology Education. Journal of Research in Science Teaching, 43 (4) 349_ 352.

Colucci-Gray, L., Camino, E., Barbiero, G. y Gray, D. (2006). From Scientific Literacy to Sustainability Literacy: An Ecological Framework for Education. Science Education, 90, $227-252$.

DeBoer, G. E. (2000). Scientific Literacy: Another Look at Its Historical and Contemporary Meanings and Its Relationship to Science Education Reform. Journal of Research in Science Teaching, 37 (6), 582-601.

España, E. (2008). Conocimiento, actitudes, creencias y valores en los argumentos sobre un tema sociocientifico relacionado con los alimentos. Tesis Doctoral. Universidad de Málaga.

España, E. y Prieto, T. (2009). Educar para la sostenibilidad: el contexto de los problemas socio-científicos. Revista Eureka sobre Enseñanza y Divulgación de las Ciencias, 6 (3), 345354.

España, E. y Prieto, T. (2010). Los problemas socio-científicos como contexto para la enseñanza y el aprendizaje de las ciencias. Investigación en la Escuela, 71, 17-24.

España, E., Prieto, T. y González, F. (2004). Juego de rol sobre los alimentos transgénicos. Un recurso didáctico CTS. En AAVV. Perspectivas Ciência-Tecnologia-Sociedade na Innovaçao em Ciencia, 301-304. Aveiro: Universidade de Aveiro.

Fourez, G. (1994). Alfabetización cientifica y tecnológica. Acerca de las finalidades de la enseñanza de las ciencias. Buenos Aires: Ediciones Colihue. Colección Nuevos Caminos.

Hodson, D. (2003). Time for action: science education for an alternative future. International Journal of Science Education, 25(6), 645-670.

Jenkins, E. W. (2009). Reforming school science education: a commentary on selected reports and policy documents. Studies in Science Education. 45(1), 65-92.

Lucas, A. (1993). Condicionantes del currículo y aportaciones de la investigación a la práctica de la educación en Ciencias. En C. Palacios, D. Ansoleaga, y A. Ajo (Eds). Diez años de investigación e innovación en enseñanza de las ciencias. Madrid: CIDE.

Martín, C. y Prieto, T. (2010). Buscando en enfoque CTS en los libros de texto de ciencias de ESO. Las Energías Renovables. Actas del II Seminario Ibero-americano de CienciaTecnología-Sociedad en la enseñanza de las ciencias. Brasilia. Brasil.

Martín, C. y Prieto, T. (2011). El potencial educativo del problema energético en la sociedad actual. En J. Maquilón, A. Mirete, A. Escarbajal y A Jiménez (Coords.). Cambios educativos y formativos para el desarrollo humano y sostenible, 29-37. Murcia: Ediciones de la Universidad de Murcia. 
Millar, R. (1996). Towards a science curriculum for public understanding. School Science Review, 77(280), 7-18.

Muñoz, E. (2002). La cultura científica, la percepción pública y el caso de la biotecnología. Ponencia presentada en el seminario: La cultura científica en la sociedad de la información. Oviedo.

Prieto, T. y España, E. (2010). Educar para la sostenibilidad. Un problema del que podemos hacernos cargo. Revista Eureka sobre Enseñaza y Divulgación de las Ciencias, 7, 216-229.

Ratcliffe, M. y Millar, R. (2009). Teaching for Understanding of Science in Context: Evidence from the Pilot Trials of the Twenty First Century Science Courses. Journal of Research in Science Teaching, 46 (8), 945-959.

Ryder, J. (2001). Identifying Science Understanding for Functional Scientific Literacy. Studies in Science Education, 36 (1), 1-44.

Schreiner, C., Henriksen, E. K. y Hansen, P. J. K. (2005). Climate Education: Empowering Today's Youth to Meet Tomorrow's Challenges. Studies in Science Education, 41, 3-50.

Simonneaux, L. (2001). Role-play or debate to promote students' argumentation and justification on an issue in animal transgenesis. International Journal of Science Education, 23 (9), 903-927.

Zeidler, D. L. y Sadler, T. D. (2008). Social and Ethical Issues in Science Education: A Prelude for Action. Science \& Education, 17, 799-803. 